FedUni ResearchOnline

http://researchonline.federation.edu.au

This is the peer-reviewed version of the following article:

Shahriyar, S., Paul, M., Murshed, M., Ali, M. (2016) Lossless hyperspectral image compression using binary tree based decomposition. 2016 International Conference on Digital Image Computing: Techniques and Applications (Dicta); Gold Coast, Australia; $30^{\text {th }}$ November $-2^{\text {nd }}$

December, 428-435.

Which has been published in final form at: http://doi.org/10.1109/DICTA.2016.7797060

Copyright (C) 2015 IEEE. Personal use of this material is permitted. Permission from IEEE must be obtained for all other uses, in any current or future media, including reprinting/republishing this material for advertising or promotional purposes, creating new collective works, for resale or redistribution to servers or lists, or reuse of any copyrighted component of this work in other works. 


\title{
Lossless Hyperspectral Image Compression Using Binary Tree Based Decomposition
}

\author{
Shampa Shahriyar, Manoranjan Paul and Manzur Murshed
}

\begin{abstract}
A Hyperspectral (HS) image provides observational powers beyond human vision capability but represents more than 100 times data compared to a traditional image. To transmit and store the huge volume of a HS image, we argue that a fundamental shift is required from the existing "original pixel intensity"based coding approaches using traditional image coders (e.g. JPEG) to the "residual" based approaches using a predictive coder exploiting band-wise correlation for better compression performance. Moreover, as HS images are used in detection or classification they need to be in original form; lossy schemes can trim off uninteresting data along with compression, which can be important to specific analysis purposes. A modified lossless HS coder is required to exploit spatial-spectral redundancy using predictive residual coding. Every spectral band of a HS image can be treated like they are individual frame of a video to impose inter band prediction. In this paper, we propose a binary tree based lossless predictive HS coding scheme that arranges the residual frame into integer residual bitmap. High spatial correlation in HS residual frame is exploited by creating large homogeneous blocks of adaptive size, which are then coded as a unit using context based arithmetic coding. On the standard HS data set, the proposed lossless predictive coding has achieved compression ratio in the range of 1.92 to 7.94 . In this paper, we compare the proposed method with mainstream lossless coders (JPEG-LS and lossless HEVC). For JPEG-LS, HEVCIntra and HEVCMain, proposed technique has reduced bit-rate by $35 \%$, $40 \%$ and $6.79 \%$ respectively by exploiting spatial correlation in predicted HS residuals.
\end{abstract}

\section{INTRODUCTION}

Hyperspectral (HS) image data are captured by sensors such as ground-based Resonon and NASA's air-based AVIRIS camera systems [1], which acquire a continuous spectrum of reflected light. Fig. 1 shows a HS image (HSI) comprising 400 to 720 nanometer $(\mathrm{nm})$ wavelength bands with $10 \mathrm{~nm}$ interval steps which also demonstrates the reflectance of different bands for different materials/objects such as soil, rock, water, and vegetation, etc.

Modern camera systems enable a HSI to include hundreds of narrow spectral bands that cover a wide range of wavelengths (300-2500nm). This wide spectrum can provide observational powers beyond human vision capabilities and has been adopted in many fields, including sugar distribution in food, food quality analysis [2], detection of chemical weapons [3], chemical composition of tablets [4], agricultural surveillance [2] and mineralogical mapping [2]. HS imaging is a non-destructive, non-contact technology which makes it ideal for a wide range of applications. HSI represents a 3D data cube with two spatial and one spectral dimensions and a HS video (HSV) can be considered a 4D data cube with time as the 4th dimension.

A HSI represents 100 (e.g., 300 bands) times more data compared to a traditional RGB (red, green, blue) image. Thus,

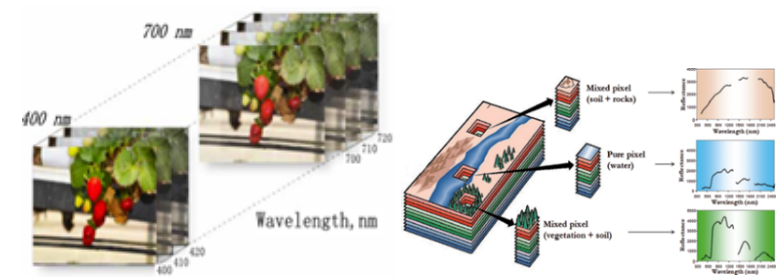

Fig. 1: A HSI with 400-720nm bands and typical reflectance [5].(Best viewed in color)

any processing and transmission of HSI needs a sophisticated compression strategy. The existing HSI lossless (where original data can be perfectly reconstructed from compressed data) compression standard [29] only provides a very small compression ratio that limits HS applications. Moreover, there is no lossy HSI compression standard as yet. We argue that a fundamental shift is required from the existing "original pixel intensity" based coding approaches using traditional image coders (e.g. JPEG-LS [6] or JPEG [7]) to the "residual" based approaches similar to a video coder (e.g. HEVC [8]) to achieve high compression ratios found with RGB images. The system can be made analogous to video coding by treating each band as a temporal frame. A high level of correlation exists in bands as they correspond to the same location of objects. However, bands corresponding to different wavelengths have different dynamic ranges, and thus, do not lend themselves easily to simple residual coding [9].

Compression schemes dedicated to hyperspectral images can be either lossy or lossless. Lossy schemes which trim off uninteresting data along with compression, are usually tailored to specific analysis purposes. Lossless schemes, on the other hand, are meant for general analysis as they preserve all raw data. In this paper, we focus on lossless schemes. The statistics of residuals in lossy (after transformation and quantization) and lossless are quite different. Moreover, the characteristics of HSI residuals are different from RGB image residuals due to their different spectral and shape domains. Consequently, existing entropy coding in HEVC is unable to provide optimum coding performance for HSI. A novel entropy coding technique is required to exploit spatial-spectral redundancy focusing on residuals. Since CABAC (contextadaptive binary arithmetic coding) [10] was originally designed for lossy texture coding, it was unable to provide the optimum coding performance for lossless hyperspectral residual image coding. A modified encoder is required to 
exploit spatial-spectral redundancy using pixel-level spectral modelling; this is due to the different characteristics of a HS image in their spectral and shape domain of panchromatic imagery, compared to traditional RGB (Red, Green and Blue) images/videos.

In this paper based on HS image property (spatially or spectral-wise highly correlated) we generate prediction frame and then using binary tree based decomposition technique we try to exploit spatial correlation in predicted residual image. Predicted nonzero residuals do not arise in isolation, instead they exhibit strong clustering tendency. Motivated by the work of Shampa et al [11], where authors tried to exploit spatial inter-component clustering tendency in depth motion vector coding using binary tree based decomposition (BTBD), in this paper a HS residual coding has been proposed using binary tree based decomposition that isolates large homogenous blocks with similar residual values. Large homogenous blocks generated from partitioning the HS residual map (on spectral level), are coded using modified context based arithmetic coding.

Experimental results on standard test dataset demonstrated that, the average compression ratio achieved by the proposed scheme was 3.67 while that achieved with other schemes were in between 2.3(JPEG-LS) to 3(HEVC). The organization of the rest of the paper is as follows. Section II presents some important literatures in HS image compression. Section III highlights basic steps of the proposed technique including residual mapping, bitmap formation, partitioning and entropy coding. Experimental results demonstrating the efficacy of the proposed scheme are presented in Section IV. Finally, the paper concludes in Section V.

\section{RELATED WORK}

Image based coders e.g. JPEG2000, in conjunction with principal component analysis (PCA) and 2D/3D discrete cosine/wavelet transform (DCT/DWT) have been widely applied in HSI compression to exploit correlations in intra-band original pixel intensity i.e. spectral reflectance. A recent study showed that 1-D Karhunen-Loeve Transform (KLT) in the spectral domain and 2D DCT/DWT in the spatial domain provides better compression [12]. However, KLT has several disadvantages such as high computational cost, large memory, bit depth expansion and lack of scalability. Blanes et al. proposed isorange pairwise orthogonal transform (IPOT) to overcome the problem of KLT [12]. Karami et al. [13] used tucker decomposition (TD) which outperforms the traditional algorithms such as PCA+JPEG2000.

CCSDS is considered a lossless HSI compression standard [29] but it does not provide the expected compression ratio due to a lack of exploitations of inter-band correlations. Gao et al. [14] used HEVC by exploiting only spatial correlation for lossy coding. Santos et al. saw the benefit using H.264 (predecessor of HEVC) [14], which outperformed image based methods where spatial and spectral correlations were exploited. Although it is a residual based approach, it could not significantly outperform existing methods due to the larger magnitude of the residuals.

Predictive coding based compression techniques employ spatial, spectral, or hybrid predictors to decorrelate the image data. In [15] the spectral redundancy in hyperspectral images is exploited using a context-match method driven by the correlation between adjacent bands. The method is suitable for hyperspectral images in the band-sequential format. However the predictors are generated using local statistics and are used based on a switching algorithm which relies on the correlation between bands solely. In [16], an inter-band version of CALIC, which adaptively selects inter-band (spectral) and intra-band (spatial) predictions, was presented. The predictor coefficients were calculated adaptively using the data correlation within the prediction context windows. This scheme was further enhanced in [17] (M-CALIC), which used only a multi-band predictor with fixed coefficients. The coefficients, however, needed to be optimized by off-line linear regression procedures.

In [18], a Kalman filter was employed to perform the prediction and the prediction residuals were coded using context based arithmetic coding. The results showed limited compression efficiency. Miguel et al. [9] showed that each band can be linearly predicted from another band. They encoded prediction coefficients and residuals. A HSI/HSV has different objects within the image, thus, the magnitude of the band-level coefficients and residuals should be larger, and thus not provide significantly better compression compared to other techniques. Moreover, it also needed reordering of bands for coding which required extra bits to inform the decoder.

\section{Proposed Method}

\section{A. Residual Generation and Mapping}

From Fig. 2 we can observe property of two different HS images Bostwana and KSC. Where Bostwana has huge variation in information and spatially more correlated, $\mathrm{KSC}$ is more simpler and spectral or band wise more correlated. So based on HSI nature intra prediction or inter-band prediction can be used. For simplicity we just used previous band as a prediction, as the main target of this work is to exploit residual correlation at band level.

Let $K$ be a band of a hyperspectral image of resolution $W \times H$. Let $\hat{k}_{i, j}$ be the predicted value of $k_{i, j}$ using intra-band or inter-band prediction. Clearly, the residual $r_{i, j}=k_{i, j}-\hat{k}_{i, j}$ can have positive and negative values in the range of $[-\hat{K}$ to $M-1-\hat{K}]$ where $M=256$ maximum pixel value. Residual ranking from CALIC [19] is used here to map the residuals into the range [0 to $M-1]$.

1) Residual map Formation: For residual map (resmap) generation we need to map the residual to non-negative values using a ranking algorithm such as Rice mapping or CALIC mapping. For context modeling another absolute residual bitmap (absresmap) will be generated from unmapped residual frame where any bitmap value at position $(i, j)$ will be $r_{i, j}^{a b s}=\left|r_{i, j}\right|$. 

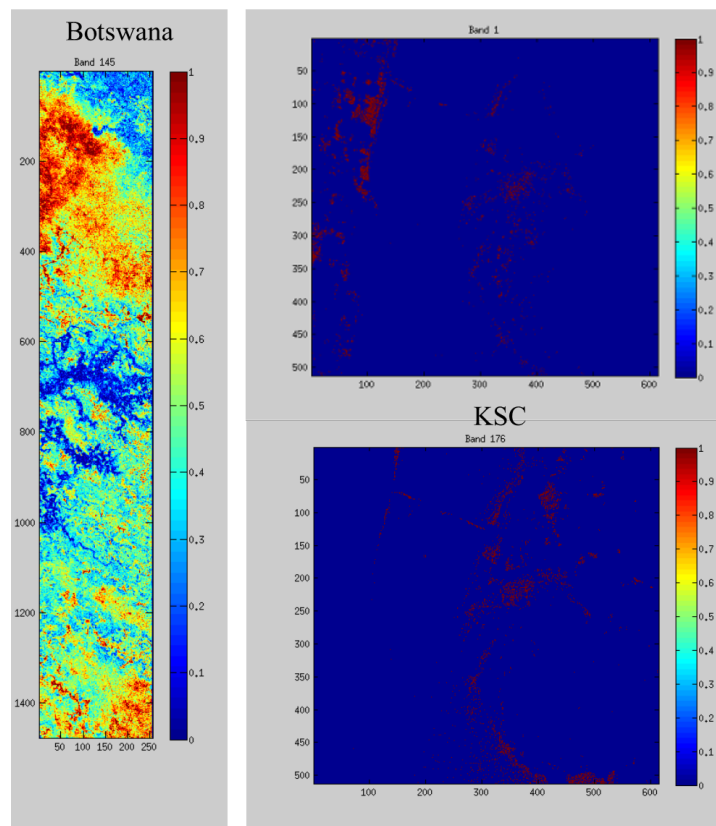

KSC

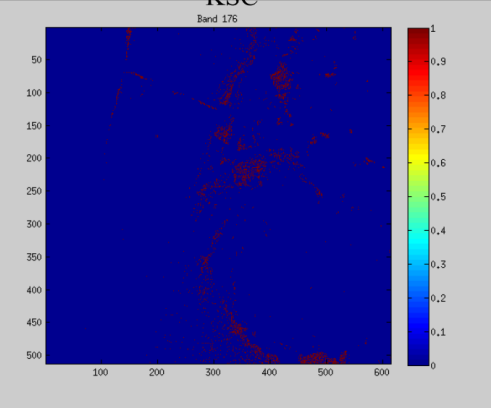

Fig. 2: Botswana and KSC(Kennedy Space Center) hyperspectral images. (Best viewed in color)

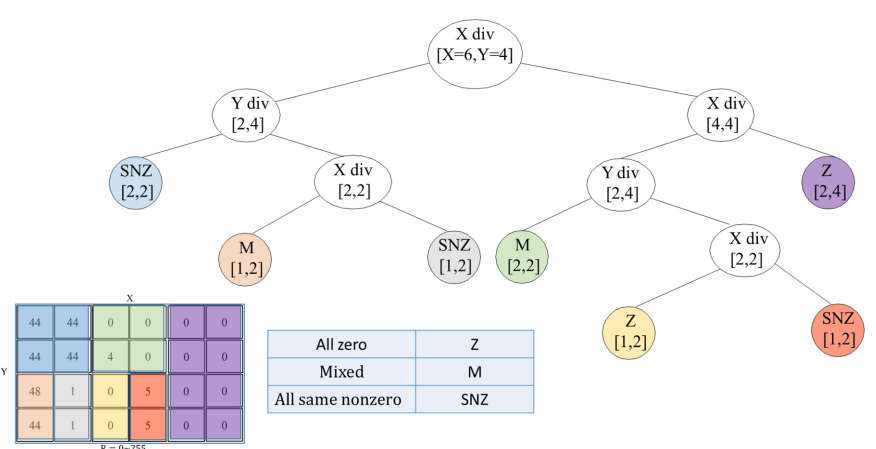

Fig. 3: Entropy based binary tree based decomposition. (Best viewed in color)

\section{B. Binary Tree Based Decomposition Using Entropy Heuristic}

1) Binary Tree Based Decomposition: After bitmap generation the proposed technique adaptively partitions the bitmaps into blocks of various dimensions. The clustering correlations are efficiently exploited during bitmap compression without any explicit run-length. Binary tree decomposition is used to adaptively partition the bitmaps of resmap into highly clustered or random blocks. Entropy heuristic has been used here to facilitate the divide-and-conquer algorithm. The division will target to divide the bitmap into sub-blocks where their entropy summation is minimized. Specifically, it tries to increase 0's or nonzero's concentration in two different blocks, therefore, fewer bits will be needed to code them. If 0's concentration is increased in one block, automatically it will increase nonzeros's concentration in another. Either it will create homogeneous blocks or stop further sub-partitioning if it is unlikely to achieve any compression. Entropy calculation will consider
TABLE I: Huffman codes of residual (codeRes)

\begin{tabular}{|c|c|c|c|}
\hline Node & Values/Division & Type & c codeRes \\
\hline \multirow{3}{*}{ Leaf } & all zero & $\mathrm{I}$ & 001 \\
\hline & all same nonzero & II & 000 \\
\hline & mixed & III & 01 \\
\hline \multirow{2}{*}{ Split } & along width & $\mathrm{X}$ & 11 \\
\hline & along height & $\mathrm{Y}$ & 10 \\
\hline
\end{tabular}

context based integer arithmetic coding cost along with context modeling cost for resmap (detailed calculation presented in Section III-C2).

The 'divide' step first checks whether the current block contains only one kind of elements, either all zero or all nonzero. In that case, the block is classified as a Type I (all zeros) or Type II (all nonzeros with same value) leaf node. Otherwise, the block is split recursively into two sub-blocks with equal size along $X$ or $Y$ axis so that less bit will be needed to code the original block. Type I requires no further information to encode. For leaf Type II, only one integer value needs to be coded for whole block.

The 'conquer' step ensures that sub-partitioning of the leaf nodes are infeasible to achieve any further compression by discarding the splitting decision made in the 'divide' step, if the zero-order entropy of the subtrees is greater than the zeroorder entropy of the current block. In that case, the block is classified as a Type III leaf node (mixed). An example of how this binary tree based decomposition technique works outlined in Figure 3.

\section{Bitmap Entropy Coder}

The type of each node of the tree is identified using the Huffman codes shown in Table I that were generated by analyzing the probability of each type in a large set of test sequences [20]. The entire tree is encoded in the pre-order depth-first traversal sequence where the root of the tree is encoded first, then recursively the left subtree, and finally, recursively the right subtree.

In lossy coding, residuals represent the quantized transform coefficients. On the contrary, in lossless coding, residuals are not the quantized transform coefficients, rather the differential pixel values between the original and predicted pixel values. Therefore, the statistics of residuals in lossy and lossless coding are quite different. Consequently, regular context based arithmetic coder has been modified here for lossless coding.

1) Context Modeling: To generate context set, the proposed technique relies on the following binarization scheme for integer residual bitmap (resmap). For any position, if $0 \leq r_{i, j} \leq 15$, then $r b_{i, j}=0$, else $r b_{i, j}=1$. Context set for resmap is as follows: $c_{1}: r b_{i, j-1}=0$ and $r b_{i-1, j}=0 ; c_{2}$ : $r b_{i, j-1}=1$ and $r b_{i-1, j}=0 ; c_{3}: r b_{i, j-1}=0$ and $r b_{i-1, j}=1$; and $c_{4}: r b_{i, j-1}=1$ and $r b_{i-1, j}=1$.

Alternatively, the proposed technique uses following contexts based on absolute residual (absresmap) value. $c_{1}$ : $0 \leq$ $r_{i, j-1}^{a b s}+r_{i-1, j}^{a b s} \leq 4 ; c_{2}: 5 \leq r_{i, j-1}^{a b s}+r_{i-1, j}^{a b s} \leq 22 ; c_{3}: 23 \leq$ $r_{i, j-1}^{a b s}+r_{i-1, j}^{a b s} \leq 107$; and $c_{4}: 108 \leq r_{i, j-1}^{a b s}+r_{i-1, j}^{a b s} \leq 512$. 
These values are adjusted empirically to maintain $\left|c_{n+1}\right| \leq$ $\frac{1}{2}\left|c_{n}\right|$.

2) Context Based Arithmetic Coder: Let $R_{c} \subseteq R$ be the set of residual values in context $c$ and $n_{c, r}$ denote the number of times residual value $r \in R_{c}$ appears in context $c$ of size $n_{c}=\sum_{r \in R_{c}} n_{c, r}$, for all $0 \leq c<C$, such that $\sum_{c=0}^{C-1} n_{c}=n$.

The context model stores the probability of each value (bin) being ' 1 ' or ' 0 '. The probability of the bin containing a 1 or a 0 is modelled based on (a) previous counts of 1 or 0 and (b) values of the same syntax element in the immediate neighborhood. The chosen probabilities are passed to an arithmetic coder which codes the bin. The context model is updated based on the actual bin contents ( 1 or 0 ).

We can encode each residual value in the block by arithmetic coding using $-\log _{2} p_{c, r}$ bits where $p_{c, r}=\frac{n_{c, r}}{n_{c}}$. is the probability of that particular residual $r$ in its context $c$. Collectively, encoding the entire block of residuals will take up at most $E_{C, R}=\left\lceil-\sum_{c=0}^{C-1} \sum_{r \in R_{c}} n_{c, r} \log _{2} p_{c, r}\right\rceil+2$ bits.

For entropy calculation to support binary tree based decomposition we need to be able to compute the same probabilities at the decoder, thus we have to compute the approximated model cost. Overhead of encoding the statistics known as model cost is $M_{C, R}=\sum_{c=0}^{C-2}\left\lceil\log _{2}\left(1+n-\sum_{j=0}^{c-1} n_{j}\right)\right\rceil+$ $\sum_{c=0}^{C-1} \sum_{r=0}^{R-2}\left\lceil\log _{2}\left(1+n_{c}-\sum_{j=0}^{r-1} n_{c, j}\right)\right\rceil$ bits. In proposed technique for integer bitmap $R=256$ and as 4 contexts have been used so $C=4$.

\section{EXPERIMENTAL RESUlt AND ANALYSis}

A lossless predictive hyperspectral image compression technique has been proposed here using binary tree based decomposition to exploit spatial and band wise correlation in HS images. For performance evaluation purpose, total bit, bit-rate (bit per pixel per band (bpppb)) and compression ratio are used as metrics ( there will be no loss in coding). Two different coding mode has been used here first, context based arithmetic coding in mapped residual image and second, binary tree based decomposition technique in mapped residual image to exploit spatial correlation in HS residual image. Two different prediction mode has been applied intra-prediction and interband prediction. Results are also presented based on different prediction modes.

The experimental results have been verified by some publicly available hyperspectral scenes [20]. All of them are earth observation images taken from airbornes or satellites. Details about dataset and their properties are described in Section IV-A. Coding performance was compared with popular lossless image coder lossless JPEG (JPEG-LS) and HEVC (Intra and Main profile using lossless condition). UBC's JPEGLS codec implementation [21] and HEVC Test Model 11 (HM 11) [22] has been used for comparison purpose. For HEVC, HS image bands are passed as a frame of video sequences with regular intra (HEVCIntra) and all mode (HEVCMain) enabled respectively. It can be observed that for the different datasets, the proposed approach consistently outperforms at a wide range of bit rates. JPEG-LS performed better than HEVCIntra as JPEG-LS is optimized for lossless image compression. The study in [23] supports this observation for natural images.

\section{A. Dataset}

1) Indian_Pines: This scene was gathered by AVIRIS sensor over the Indian Pines test site in North-western Indiana and consists of $145 \times 145$ pixels and 220 spectral reflectance bands in the wavelength range $0.42 .5 \times 10^{-6}$ meters. This scene is a subset of a larger one. The Indian Pines scene contains two-thirds agriculture, and one-third forest or other natural perennial vegetation.

2) Salinas scene: This scene was collected by the 224band AVIRIS sensor over Salinas Valley, California, and is characterized by high spatial resolution (3.7-meter pixels). The area covered comprises 512 lines by 217 samples. It includes vegetables, bare soils, and vineyard fields. Salinas-A scene is a small subscene of Salinas image, denoted Salinas-A, is usually used too. It comprises $86 \times 83$ pixels located within the same scene.

3) Pavia Centre and University: These are two scenes acquired by the ROSIS sensor during a flight campaign over Pavia, nothern Italy. The number of spectral bands is 102 for Pavia Centre and 103 for Pavia University. Pavia Centre is a $1096 \times 1096$ pixels image, and Pavia University is $610 \times 610$ pixels, but some of the samples in both images contain no information and have to be discarded before the analysis.

4) Airborne Visible/Infrared Imaging Spectrometer (AVIRIS): Cuprite: The dataset used in this part of the experiment is from Airborne Visible/Infrared Imaging Spectrometer (AVIRIS) reflectance data. AVIRIS data are mainly collected for identifying, measuring, and monitoring constituents of the earth's surface and atmosphere based on molecular absorption and particle scattering signatures. It delivers calibrated images with 206 contiguous spectral bands and approximately $10 \mathrm{~nm}$ spectral resolutions covering the $0.4-2.5 \mu \mathrm{m}$ spectral range. The data was collected from mineral mapping at Cuprite, Nevada.

5) Kennedy Space Center (KSC): The NASA AVIRIS (Airborne Visible/Infrared Imaging Spectrometer) instrument acquired data over the Kennedy Space Center (KSC), Florida, on March 23, 1996. AVIRIS acquires data in 224 bands of $10 \mathrm{~nm}$ width with center wavelengths from $400-2500 \mathrm{~nm}$. The KSC data, acquired from an altitude of approximately 20 $\mathrm{km}$, have a spatial resolution of $18 \mathrm{~m}$. After removing water absorption and low SNR bands, 176 bands were used for the analysis.

6) Botswana: The NASA EO-1 satellite acquired a sequence of data over the Okavango Delta, Botswana in 20012004. The Hyperion sensor on EO-1 acquires data at $30 \mathrm{~m}$ pixel resolution over a $7.7 \mathrm{~km}$ strip in 242 bands covering the 400-2500 nm portion of the spectrum in $10 \mathrm{~nm}$ windows. Preprocessing of the data was performed by the UT Center for Space Research to mitigate the effects of bad detectors, inter-detector miscalibration, and intermittent anomalies. Uncalibrated and noisy bands that cover water absorption features 


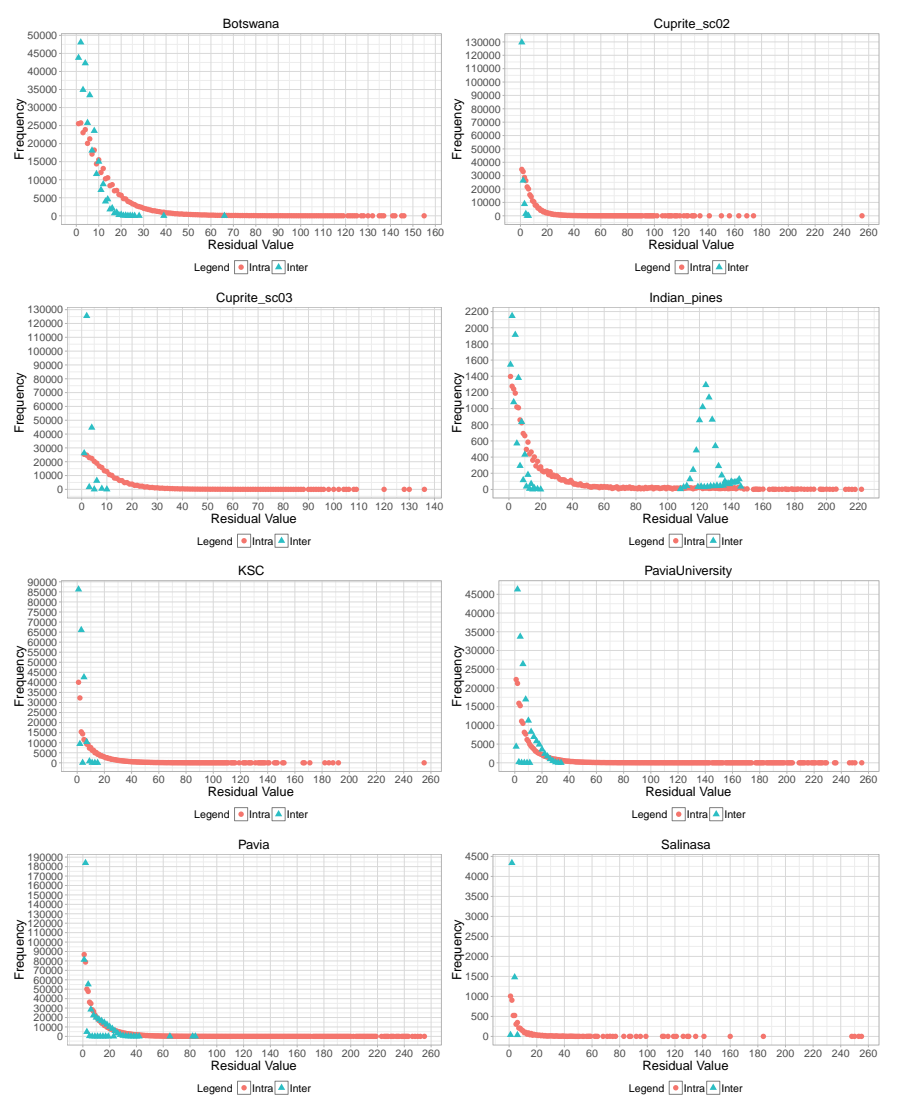

Fig. 4: Predicted residual value analysis for hyperspectral images (Band 76 for all sequences). (Best viewed in color)

were removed, and the remaining 145 bands were included as candidate features.

Fig. 4 exhibits the frequency of zero and non-zero values in predicted residual of HS images for a particular band using intra and inter-band prediction. It is observable that simple inter-band predicted HS residual contains less variation in values and zero values are majority, which implies band-wise correlation in HS images. Figure 5 and 6 illustrate the original band and intra and inter band predicted residual of Pavia University and KSC dataset. It clearly shows the strong spatial correlation in predicted residual (specially in inter prediction) that zero and non-zero values appeared in clustered way. Using binary tree based decomposition this correlation can be exploited by creating large blocks of same residual values.

\section{B. Results and Analysis}

Table II presents the compression performance (total bit and compression ratio $(\tau)$ ) of JPEG-LS, HEVC Intra and Main, proposed technique with intra and inter band prediction for all the test dataset. Other than Salinas_A and KSC proposed BTBD technique with inter-band prediction outperforms other techniques with better compression ratio. Proposed lossless technique achieves compression ratio as high as 7.94 and on average 3.67. Table III presents the coding gain of proposed technique by bit-rate reduction percentage. Moreover it sum- maries the overall bit-rate of proposed technique in bit per pixel per band. For JPEG-LS proposed technique has reduced bit-rate by $35 \%$ and for HEVCIntra this is $40 \%$. HEVCMain with lossless condition by exploiting inter-band correlation in specific coding order outperformed proposed technique for two dataset with $22 \%$ and $1 \%$ bit-rate gain. Overall proposed technique outperforms HEVCMain with $6.79 \%$ of bit reduction by exploiting spatial correlation in predicted HS residuals.

Fig. 7 and 8 illustrate the division of HS residual map (for band 76) using proposed binary tree based decomposition for PaviaUniversity and KSC dataset. For PaviaUniversity the binary tree division contains 12098 1D and 256 2D leaf nodes (total HS band size is 207400 pixels) where average leaf nodes size is 17 pixels and as high as 399 pixels. For KSC dataset, binary tree division contains 106 1D and 2648 2D leaf nodes (total HS band size is 314368 pixels) where average and maximum leaf node size is 114 and 4992 pixels. This exhibits the efficacy of proposed division based residual coding technique. Computational complexity of any tree decoding is of the order of the leaf nodes in the tree. Therefore, the complexity overhead of the decoder is of little concern.

\section{CONCLUSION}

Whereas the human eye sees color of visible light in mostly three bands (red, green, and blue), spectral imaging divides the spectrum into many more bands. This technique of dividing images into bands can be extended beyond the visible. Engineers build hyperspectral sensors and processing systems for applications in astronomy, agriculture, biomedical imaging, geosciences, physics, and surveillance. For HS as a third dimension is added, the amount of data increases dramatically making the compression necessary at different steps of the processing chain. Moreover, the high expectations from the scientists using hyperspectral data require the assurance that the compression will not degrade the data quality, which raises the issue of efficient lossless HS compression.

Where for regular image coding original pixel based coding approaches are used but for HS images "residual" based approaches using a predictive coder is needed to exploit bandwise correlation for better compression performance. In this paper, we proposed a lossless HS coder to exploit spatial and

TABLE III: Bit-rate gain(\%) by proposed technique. Here (-) negative value denotes bit-rate reduction.

\begin{tabular}{|c|c|c|c|c|}
\hline \multirow{2}{*}{ Sequence } & \multirow{2}{*}{ Proposed(bpppb) } & \multicolumn{3}{|c|}{ Bit-rate Gain $(\%)$} \\
\hline & & JPEG-LS & HEVCIntra & HEVCMain \\
\hline Botswana & 2.99 & -31.29 & -42.74 & -25.31 \\
\hline Cuprite_sc02 & 2.37 & -46.79 & -62.99 & -16.37 \\
\hline Cuprite_sc03 & 2.87 & -39.78 & -57.02 & -11.77 \\
\hline Indian_pines & 4.17 & -22.04 & -32.19 & -9.82 \\
\hline $\mathrm{KSC}$ & 1.01 & -33.16 & 22.01 & 22.02 \\
\hline Pavia & 1.97 & -52.53 & -58.79 & -6.47 \\
\hline PaviAU & 2.08 & -51.38 & -57.60 & -7.78 \\
\hline Salinas_A & 2.96 & -9.83 & -32.18 & 1.15 \\
\hline \multicolumn{2}{|c|}{ Average } & -35.85 & -40.19 & -6.79 \\
\hline
\end{tabular}




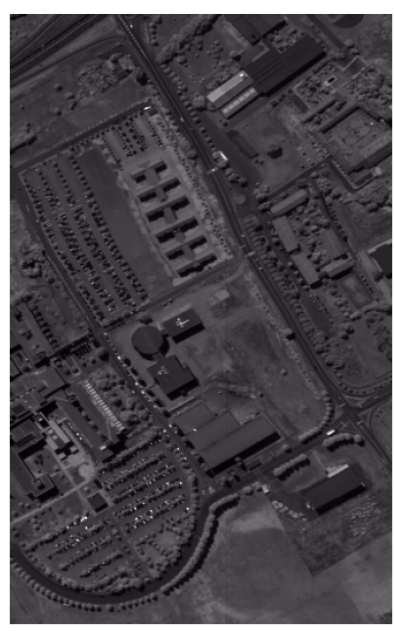

(a) Original Band 76

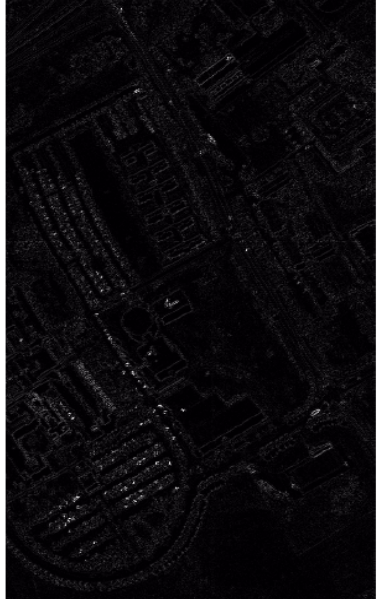

(b) Intra predicted residual of band 76

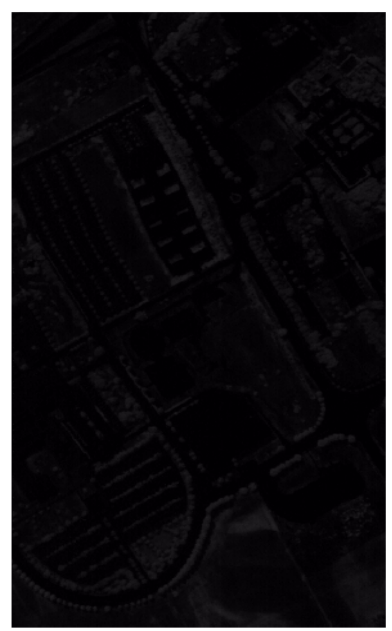

(c) Inter predicted residual of band 76

Fig. 5: Original Band 76 with predicted residual for Pavia University test sequence.(Best viewed in color)

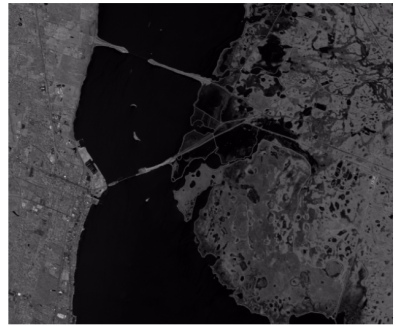

(a) Original Band 76

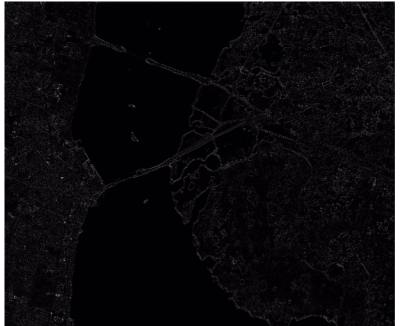

(b) Intra predicted residual of band 76

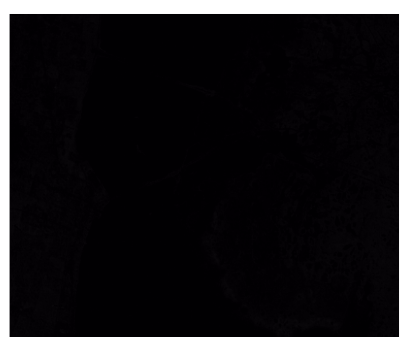

(c) Inter predicted residual of band 76

Fig. 6: Original Band 76 with predicted residual for KSC test sequence. (Best viewed in color)

TABLE II: Comparison of Compression Ratio $(\tau)$

\begin{tabular}{|c|c|c|c|c|c|c|c|c|c|c|c|c|c|}
\hline \multirow{2}{*}{ Sequence } & \multirow{2}{*}{ Width } & \multirow{2}{*}{ Height } & \multirow{2}{*}{ Band } & \multicolumn{2}{|c|}{ JPEG-LS } & \multicolumn{2}{|c|}{ HEVCIntra } & \multicolumn{2}{|c|}{ HEVCMain } & \multicolumn{2}{|c|}{ IntraBTBD } & \multicolumn{2}{|c|}{ InterBTBD } \\
\hline & & & & bit & $\tau$ & bit & $\tau$ & bit & $\tau$ & bit & $\tau$ & bit & $\tau$ \\
\hline Botswana & 1476 & 256 & 145 & 238371528 & 1.84 & 286056064 & 1.53 & 219309216 & 2.00 & 236250608 & 1.86 & 163796036 & 2.68 \\
\hline Cupritesc 02 & 512 & 614 & 206 & 287169936 & 1.80 & 412871004 & 1.25 & 182708124 & 2.84 & 284551975 & 1.82 & 152807435 & 3.39 \\
\hline Cupritesc 03 & 512 & 614 & 206 & 308439328 & 1.68 & 432149532 & 1.20 & 210528148 & 2.46 & 303369175 & 1.71 & 185741977 & 2.79 \\
\hline Indianpines & 145 & 145 & 220 & 24746768 & 1.50 & 28451952 & 1.30 & 21394736 & 1.73 & 24896467 & 1.49 & 19293495 & 1.92 \\
\hline KSC & 512 & 614 & 176 & 83375200 & 5.31 & 45677448 & 9.69 & 45672872 & 9.69 & 90137235 & 4.91 & 55730143 & 7.94 \\
\hline Pavia & 1096 & 715 & 102 & 331810592 & 1.93 & 382237730 & 1.67 & 168412498 & 3.80 & 340976929 & 1.88 & 157516001 & 4.06 \\
\hline PaviAU & 610 & 340 & 103 & 91212472 & 1.87 & 104595330 & 1.63 & 48086250 & 3.55 & 92837125 & 1.84 & 44347227 & 3.85 \\
\hline SalinasA & 83 & 86 & 204 & 4779400 & 2.44 & 6353988 & 1.83 & 4260228 & 2.73 & 5695565 & 2.05 & 4309350 & 2.70 \\
\hline
\end{tabular}

band-wise correlation of HS images using predictive coding and later to exploit the high correlation within HS residuals binary tree based decomposition technique has been incorporated. The division will target to generate large homogenous adaptive size blocks which can be coded as a whole using context based arithmetic coding. Where for natural images, lossless coders like JPEG-LS, lossless HEVC give compression ratio between 1-3, but proposed technique has achieved compression ration as high as 7.94 and on average 3.67 for HS images. Moreover, for encoding most of the HS data-set, bit needed per pixel per band is within 1 to 2 , which is quite low compared to other lossless HS compression techniques.
By exploiting spatial and spectral wise correlation among HS bands and spatial smoothness between HS residuals on band level, for the standard data set, proposed technique has reduced bit-rate by $35 \%, 40 \%$ and $6.79 \%$ compared to JPEGLS, HEVCIntra and HEVCMain coders respectively.

\section{REFERENCES}

[1] “Aviris camera systems," retrieved 28th July 2015. [Online]. Available: http://aviris.jpl.nasa.gov/ [1]

[2] D. Wu and D.-W. Sun, "Advanced applications of hyperspectral imaging technology for food quality and safety analysis and assessment: A review part i: Fundamentals," Innovative Food Science and Emerging Technologies, vol. 19, pp. $1-14$, 2013. [1] 


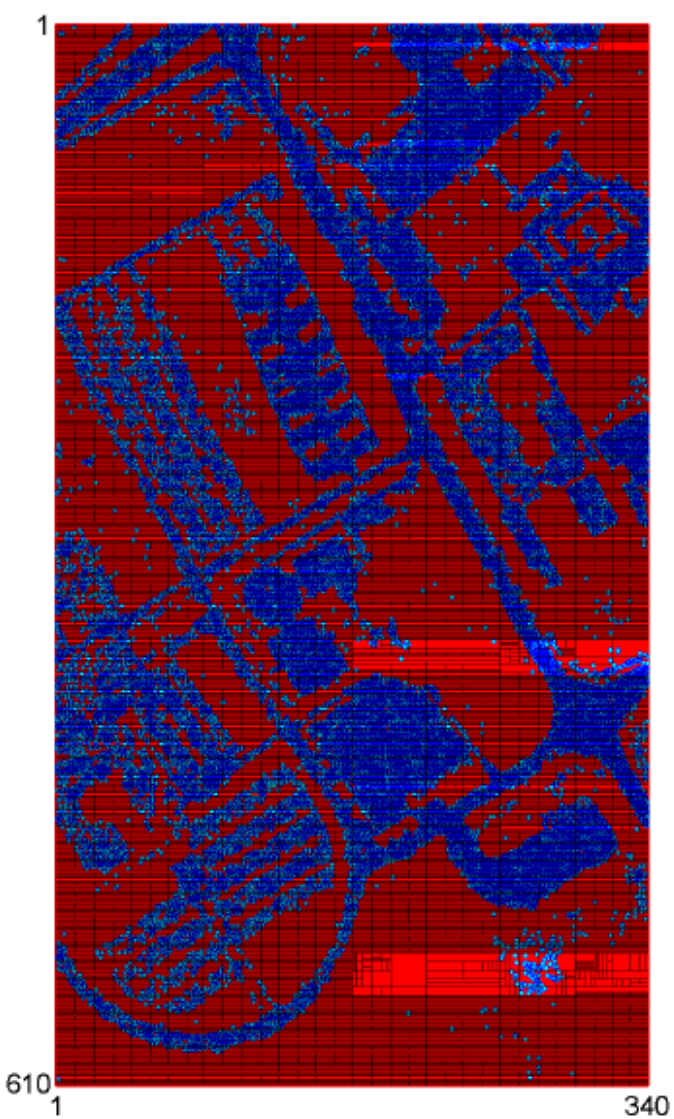

Fig. 7: Spatial Correlation exploitation in predicted residual of HS image (Pavia University band 76) using binary tree based decomposition. Here blue and red dots indicate zero and nonzero residual values respectively. (Best viewed in color)

[3] M. Dao, D. Nguyen, T. Tran, and S. Chin, "Chemical plume detection in hyperspectral imagery via joint sparse representation," in MILCOM 2012 - 2012 IEEE Military Communications Conference, Oct 2012, pp. $1-5 .[1]$

[4] A. Palou, J. Cruz, M. Blanco, J. Toms, J. de los Ros, and M. Alcal, "Determination of drug, excipients and coating distribution in pharmaceutical tablets using nir-ci," Journal of Pharmaceutical Analysis, vol. 2, no. 2, pp. 90 - 97, 2012. [1]

[5] "Reflectance distribution for different objects in different wavelength," retrieved 15 July 2015. [Online]. Available: http://remotesensing. spiedigitallibrary.org [1]

[6] M. Weinberger, G. Seroussi, and G. Sapiro, "From LOGO-I to the JPEG-LS standard," in Proceedings of IEEE International Conference on Image Processing (ICIP), vol. 4, 1999, pp. 68-72. [1]

[7] G. Wallace, "The JPEG still picture compression standard," IEEE Trans. on Consumer Electronics, vol. 38, no. 1, pp. xviii-xxxiv, Feb 1992. [1]

[8] G. J. Sullivan, J. R. Ohm, W. J. Han, and T. Wiegand, "Overview of the high efficiency video coding (hevc) standard," IEEE Transactions on Circuits and Systems for Video Technology, vol. 22, no. 12, pp. 16491668, Dec 2012. [1]

[9] A. C. Miguel, R. E. Ladner, E. A. Riskin, S. Hauck, D. K. Barney, $\ddagger$ R. Askew, and A. Chang, Predictive Coding of Hyperspectral Images. Boston, MA: Springer US, 2006, pp. 197-231. [1, 2]

[10] D. Marpe, H. Schwarz, and T. Wiegand, "Context-based adaptive binary arithmetic coding in the H.264/AVC video compression standard," IEEE Trans. Circuits Syst. Video Technol., vol. 13, no. 7, pp. 620-636, July 2003. [1]

[11] S. Shahriyar, M. M. Murshed, M. Ali, and M. Paul, "A novel depth

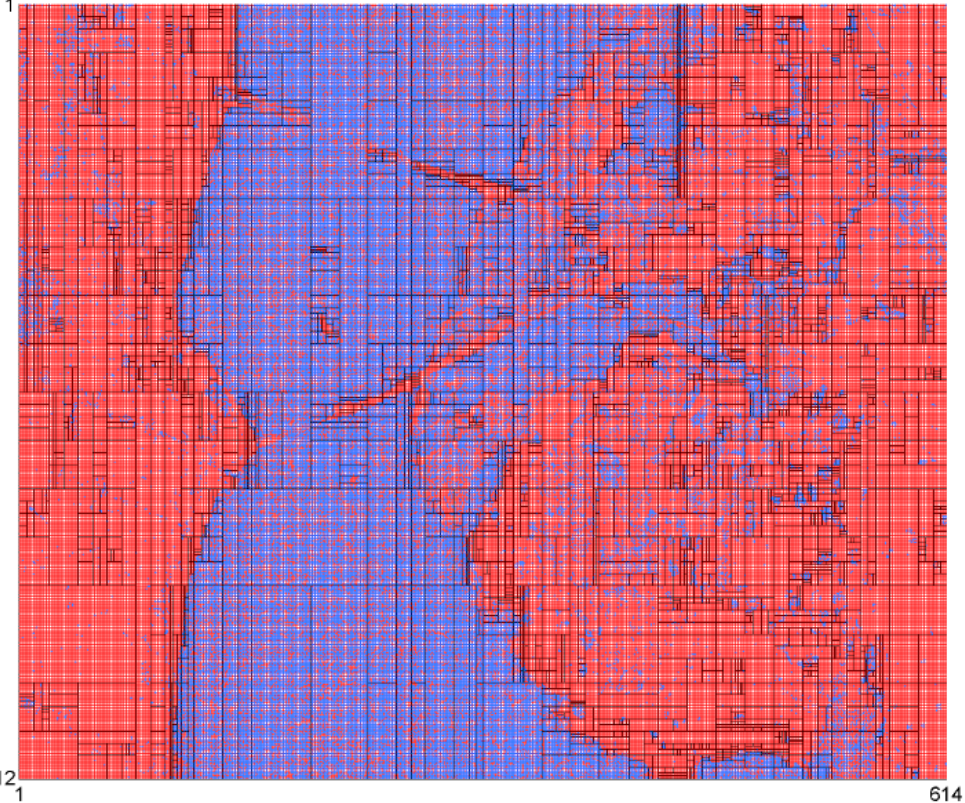

Fig. 8: Spatial Correlation exploitation in predicted residual of HS image (KSC band 76) using binary tree based decomposition. Here blue and red dots indicate zero and non-zero residual values respectively. (Best viewed in color)

motion vector coding exploiting spatial and inter-component clustering tendency," in 2015 Visual Communications and Image Processing, VCIP 2015, Singapore, December 13-16, 2015, 2015, pp. 1-4. [2]

[12] I. Blanes, M. Hernndez-Cabronero, F. Aul-Llins, J. Serra-Sagrist, and M. W. Marcellin, "Isorange pairwise orthogonal transform," IEEE Transactions on Geoscience and Remote Sensing, vol. 53, no. 6, pp. 33613372, June 2015. [2]

[13] A. Karami, M. Yazdi, and G. Mercier, "Compression of hyperspectral images using discerete wavelet transform and tucker decomposition," IEEE Journal of Selected Topics in Applied Earth Observations and Remote Sensing, vol. 5, no. 2, pp. 444-450, April 2012. [2]

[14] L. Santos, S. Lopez, G. M. Callico, J. F. Lopez, and R. Sarmiento, "Performance evaluation of the h.264/avc video coding standard for lossy hyperspectral image compression," IEEE Journal of Selected Topics in Applied Earth Observations and Remote Sensing, vol. 5, no. 2, pp. 451-461, April 2012. [2]

[15] H. Wang, S. D. Babacan, and K. Sayood, "Lossless hyperspectralimage compression using context-based conditional average," IEEE Transactions on Geoscience and Remote Sensing, vol. 45, no. 12, pp. 4187-4193, Dec 2007. [2]

[16] X. Wu and N. Memon, "Context-based lossless interband compressionextending calic," IEEE Transactions on Image Processing, vol. 9, no. 6 , pp. 994-1001, Jun 2000. [2]

[17] E. Magli, G. Olmo, and E. Quacchio, "Optimized onboard lossless and near-lossless compression of hyperspectral data using calic," IEEE Geoscience and Remote Sensing Letters, vol. 1, no. 1, pp. 21-25, Jan 2004. [2]

[18] E. Magli, "Multiband lossless compression of hyperspectral images," IEEE Transactions on Geoscience and Remote Sensing, vol. 47, no. 4, pp. 1168-1178, April 2009. [2]

[19] X. Wu and N. Memon, "Calic-a context based adaptive lossless image codec," in IEEE International Conference on Acoustics, Speech and Signal Processing (ICASSP), vol. 4, May 1996, pp. 1890-1893 vol. 4. [2]

[20] "Hyperspectral Remote Sensing Scenes," retrieved 28th July 2016 [Online]. Available: http://www.ehu.eus/ccwintco/index.php?title= Hyperspectral_Remote_Sensing_Scenes [3, 4] 
[21] U. J.-L. C. Implementation, "JPEG-LS the emerging lossless/nearlossless compression standard for continuous-tone still images software," [Online; accessed 30-September-2016]. [4]

[22] "High efficiency video coding (HEVC) test model 11 (HM 11)," retrieved 28th July 2016. [Online]. Available: https://hevc.hhi.fraunhofer de/svn/svn_HEVCSoftware/tags/HM-11.0/ [4]

[23] Q. Cai, L. Song, G. Li, and N. Ling, "Lossy and lossless intra coding performance evaluation: Hevc, h. 264/avc, jpeg 2000 and jpeg ls," in Signal and Information Processing Association Annual Summit and Conference (APSIPA ASC), 2012 Asia-Pacific. IEEE, 2012, pp. 1-9. [4] 